\title{
Establishing an information security strategy
}

\author{
Erik Guldentops CISA \\ Director of Global Information Security \\ S.W.I.F.T. (Society for Worldwide Interbank Financial \\ Telecommunication) \\ Avenue Adèle 1 \\ B-1310 La Hulpe \\ Belgium \\ tel.: +3226553111 \\ fax: +3226553226
}

\begin{abstract}
Security is seen as fundamental to S.W.I.F.T.'s business, second only to network availability. The security infrastructure in terms of organisation, direction, processes and tools, must be appropriate. The world as we know it is changing rapidly as technology marches forward and as the modern business world expands to take advantage of the new technology. S.W.I.F.T. intends to face these challenges head on. This document introduces the company's strategic plans in the important area of Security and outlines six new projects which will commence this year.
\end{abstract}

\section{Keywords}

SWIFT, financial transactions, security architecture, security awareness, public key infrastructure, security strategy, policies, standards, access control, risk assessment, business process security

\section{INTRODUCTION}

The world as we know it is changing rapidly as technology marches forward and as the modern business world expands to take advantage of the new technology. The company intends to face these challenges head on. There is simply no alternative. New products and services are being introduced which are different, in many ways, 
from the traditional FIN-type services. Our security infrastructure in terms of organisation, direction, processes and tools, must be appropriate and relevant to these new opportunities.

This document introduces S.W.I.F.T.'s strategic plans in the important area of Security and outlines six new projects which will commence this year.

From the organisational point of view, Security is fundamental to S.W.I.F.T.'s success:

- S.W.I.F.T.'s members have a legal obligation to secure their customer's funds. S.W.I.F.T. acts as an agent of the members, and therefore must also protect the member's customer's funds.

- The members must be able to trust S.W.I.F.T. and have stated, in the third article of S.W.I.F.T.'s charter, that the services provided must be secure.

- The amount of funds being transmitted are vast and would be a very attractive target for well funded and technically advanced criminal organisations, hackers, disgruntled S.W.I.F.T. staff, etc. Both the value of the risks, and therefore the need for security are increasing, while the required knowledge to perpetrate an attack is decreasing.

- S.W.I.F.T. has a unique R\&L Policy. Inadequate security could allow significant attack which, via the R\&L, could lead to major financial losses.

- S.W.I.F.T. also has a unique Trusted Third Party position, based on its established credibility. The damaging effect of a perceived penetration into the S.W.I.F.T. system would be dramatic on S.W.I.F.T.'s credibility.

- Security is one of the major differentiators that S.W.I.F.T. offers. Without security S.W.I.F.T. would have many competitors, losing market share and revenue.

Similarly, in every customer survey, Security is seen as fundamental to S.W.I.F.T.'s business, second only to network availability. But, powerful market forces exist, driving our business and the technology on which it is based, into a new - and often hostile - areas:

- Traditional strengths: Bandwidth, network connectivity and messaging standards - once unique to S.W.I.F.T. - are fast becoming commodities which are widely available. The desire to support 'Open' systems and protocols (such as UNIX and TCP/IP) undermines existing security mechanisms. Centralised computer systems are being replaced by client-server architectures, allowing servers to be distributed, with remote clients connecting via third-party networks. S.W.I.F.T. will evolve from offering a communications infrastructure, to offering:

- secure managed services, accessed in an open public network environment

- a forum for members to establish standards enabling them to do business.

- New business requirements: There is a clear demand for new and more complex financial messaging services:

- Interactive Services - offering one-to-one query-response facilities 
- Directory Services - where ' $n$ ' users place information in a central repository, to be accessed by ' $n$ ' different users

- Infrastructure Projects - for Central Banks and for Clearing Houses

- Electronic Commerce - involving international trading via the Internet, where there is an opportunity for S.W.I.F.T. to act as the Trusted Third Party for the parties involved

- Electronic Publishing - providing Web-type information services.

- Modular R\&L: S.W.I.F.T. is expected to extend the bounds of its Responsibility and Liability beyond the SAP and up to the CBT, and many users are positioning their CBTs deep within their own networks. Smaller users want S.W.I.F.T. to manage their CBT, while larger users do not. This requires a far more modular approach to the concept of R\&L.

- The Internet: While this all-invasive technology can provide reliable, highspeed, low cost and worldwide communications, it remains wholly unregulated, inherently insecure and represents both a hackers paradise and an active breeding ground for viruses.

To meet the expectations of our customers and to fulfil our corporate mission, a new security architecture has been sought that will enable S.W.I.F.T. to retain - and indeed enhance - the security of its products, applications and financial messaging services while, at the same time, being able to take advantage of the new business opportunities and today's open technologies.

S.W.I.F.T. has carried out a detailed review of its security and has now defined an enhanced Security Strategy for the next five years. Six new security-related projects are summarised here; the remaining sections of this document provide further details on each of these projects.

\section{THE SECURITY STRATEGY DEVELOPMENT PROCESS}

Throughout 1996, S.W.I.F.T. was engaged in a detailed process of consultation and planning in support of the company's future business requirements, so as to define the necessary generic security requirements.

These security requirements have to embrace the rapidly-changing world, where both S.W.I.F.T. and its customers must operate in an increasingly hostile communications environment. 


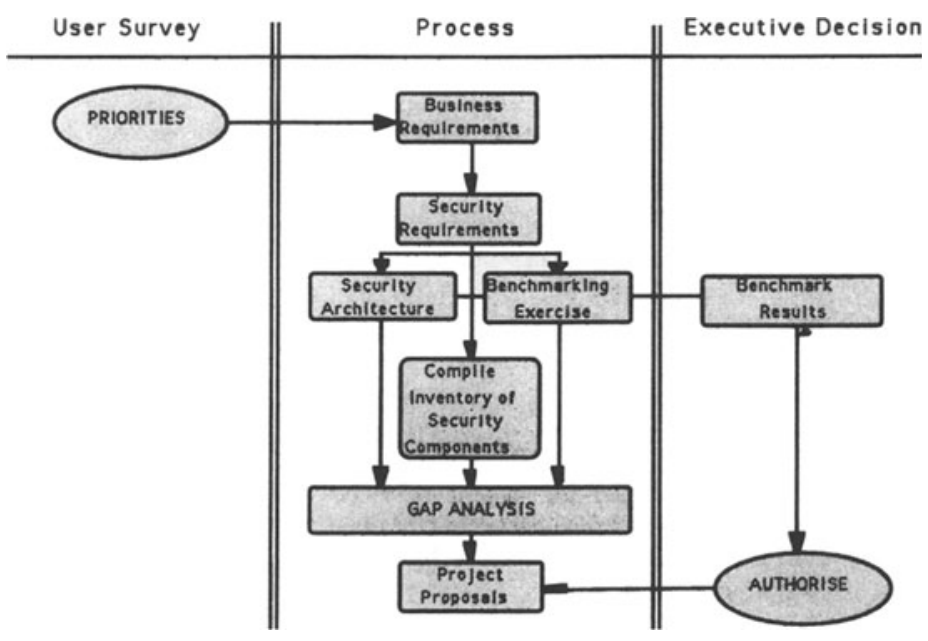

Figure 1. Security Strategy Development process

\section{Security Architecture and Strategy}

A Security Architecture was designed as a framework within which a clear Security Strategy was defined. The Security Strategy is an enabler to the financial business.

This architecture is rather like a house, where the fundamental requirements of the business are supported by a defined Security Strategy, detailing the security services that need to be offered, the security mechanisms employed and the tools and technology to be used, together with the necessary security management controls. Importantly, all of this must rest on a secure foundation of strong internal company-wide security practices and procedures, designed to create and to maintain a security-minded culture within the company.

The company's new Security Strategy aims to enable S.W.I.F.T. to achieve its primary corporate mission: "to be the recognised leader in global secure financial communications." 


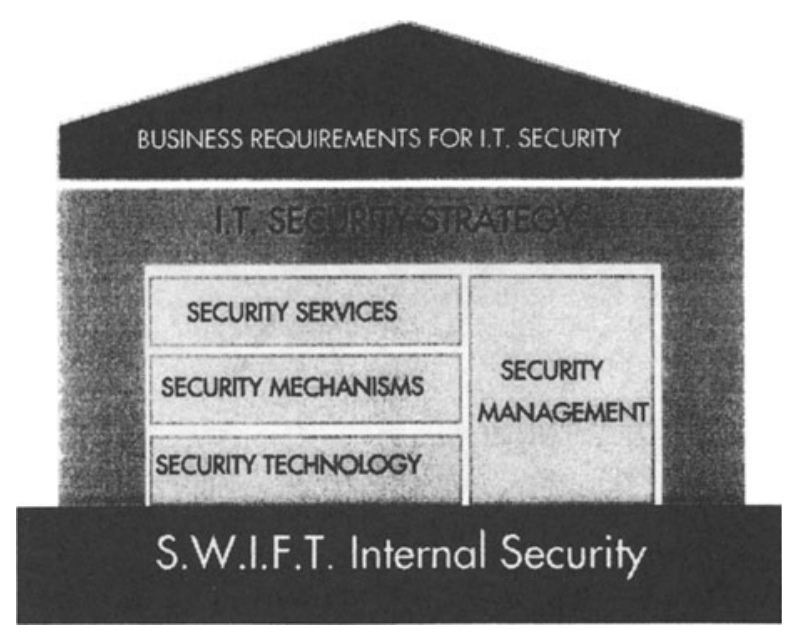

Figure 2. Security Architecture and Strategy

This strategy recognises that S.W.I.F.T. is not in the security business per se, but in the business of secure financial messaging services. It enshrines the following principles:

- Security can never be an optional or additional facility; it must be intrinsic within our culture and embedded at the heart of our services, applications, interfaces and communications.

- Security must be truly end-to-end, no longer just within the network domain.

- Solutions must be multi-tiered: based on software, and on smart cards and on hardware, according to users' requirements.

- Risks must be identified, protected against and underwritten and all security mechanisms must be certified.

- The security we offer our customers must be designed and built with performance in mind, using state-of-the-art technology and be modular, flexible and scalable.

- Security mechanisms must be independent of applications and connectivity, and be relevant to our ever more diversified customer base.

Above all the security mechanisms must be user-friendly and unobtrusive - based on 'plug-and-play' principles - and be capable of being implemented at reasonable cost, with a smooth and well-defined transition path. 


\section{Benchmarking Methodology}

To quantify our security, S.W.I.F.T. has developed a formal benchmarking methodology in order to measure - in a truly objective manner - our strengths and weaknesses. Users, security experts, Standards bodies and Auditors were consulted and a complete inventory of all systems and security components within the company has been compiled. In each of the many security domains identified, differing levels of achievement were strictly defined and a scoring system devised. The weighted aggregate of all scores enables a corporate-wide 'Security Performance Indicator' (SPI) to be calculated.

Importantly the SPI enables us to determine where we are today, to set measurable targets for where we wish to be in the future, to monitor improvements year by year and to compare S.W.I.F.T.'s security-related performance with the rest of the financial industry and with international standards. The SPI also provides clear evidence to customers of S.W.I.F.T.'s commitment to IT security and leadership in the provision of secure financial services. This methodology has now been fully documented and is available to customers who wish to conduct a similar exercise.

\section{Analysis}

By performing a Gap Analysis using the above results, S.W.I.F.T. has been able to identify potential risks and weaknesses and so determine precisely what has to be done in order to achieve the planned excellence in security. A total of 34 security domains were defined, documented and benchmarked during the Security Strategy Development Process. Six key security projects have been defined within the areas of Policy and Procedures, Processes and Tools and Technology. Each project will play a major part in the implementation of S.W.I.F.T.'s security strategy and, therefore, in helping to enable S.W.I.F.T. to achieve its strategic business objectives.

\section{THE RESULTS...}

The following six projects form an essential part of the SWIFT2001 strategic plan and have been fully endorsed by both the Executive Management and by the Board. Budgets and manning have been approved for 1997 and the message is clear security is one of the most important aspects of S.W.I.F.T.'s business and, by investing heavily in its global infrastructure, SWIFT does indeed intend to lead the world in all aspects of IT security and in secure global financial communications.

\section{Policies and Procedures}

S.W.I.F.T. plans to implement detailed security policies and procedures following the 1996 release of the Corporate Security Policy and Corporate Security Standards. 


\section{Security Management}

This project will improve security management controls and responsiveness to Security Audit recommendations. S.W.I.F.T. will provide ongoing security solutions for all new products and for the new internal infrastructure, including the provision of secure and controlled Internet access. Security administration of internal systems will be improved through the deployment and activation of new security tools.

\section{Human Behaviour and Culture}

The security culture within S.W.I.F.T. will be improved by rolling out a continuous Security Awareness programme for all staff, by developing a generic Security Guide for customers and by instituting a UNIX Security Certification programme for technical staff.

\section{Application Security and Public Key Infrastructure}

S.W.I.F.T. will select the vendor and begin the roll-out of a software-based Public Key Infrastructure (PKI) as the basis of a new secure communications infrastructure. Initial deliverables for this major project will include a digital signature tool for the Value Added Systems and security tools for Board Communications. Also a feasibility study will be performed for a 'lite' version of USE, so as to most easily support a diversifying customer base.

\section{System Access Controls}

S.W.I.F.T. plans to implement a central database for the management of all access controls and will initiate the development of the required software to connect the various applications to this central system. S.W.I.F.T. will strengthen user authentication by piloting the development of access controls based on biometrics (e.g. fingerprint recognition) initially for the new Customer Support System.

\section{Network Segregation}

This major project will see the S.W.I.F.T. networks segregated into three distinct domains: Production, Support/Management, and Office Automation. These will be protected from each other and from the external world, by building and implementing appropriate Firewall systems. This new network topology will also enable secure use of emerging and efficient communication means, such as TCP/IP and the Internet. 


\section{POLICIES AND PROCEDURES}

In order to establish and to enforce the S.W.I.F.T. security framework, the company plans to implement detailed security policies, standards, practices and guidelines and to publish these to all concerned. These documents will form the basis for all security activities within the company and will make an important contribution to improving S.W.I.F.T.'s security culture.

Much of this work has already been accomplished and the goal for the early part of 1997 is to ensure that detailed security procedures exist, supported by an asset classification scheme. Thereafter, S.W.I.F.T. aims to achieve a fully integrated and structured set of procedures, which are effectively applied.

\section{Definitions}

POLICY: High level executive statements on how the organisation must operate from a security point-of-view, stating purpose, objectives and responsibilities.

STANDARDS: The generic techniques, measures and organisational structures designed to provide reasonable assurance that the security objectives will be achieved and that risks will be minimised.

PRACTICES AND BASELINES: Detailed subsets of techniques, measures and organisational structures which form a coherent whole for a given area of application. The level of detail and coherence will be such that these practices and baselines will form the basis for efficient and effective implementation by those responsible for the domain, process, system or application.

PROCEDURES: Step-by-step details for the implementation of the Practices, relevant to a specific domain of responsibility, process, system or application.

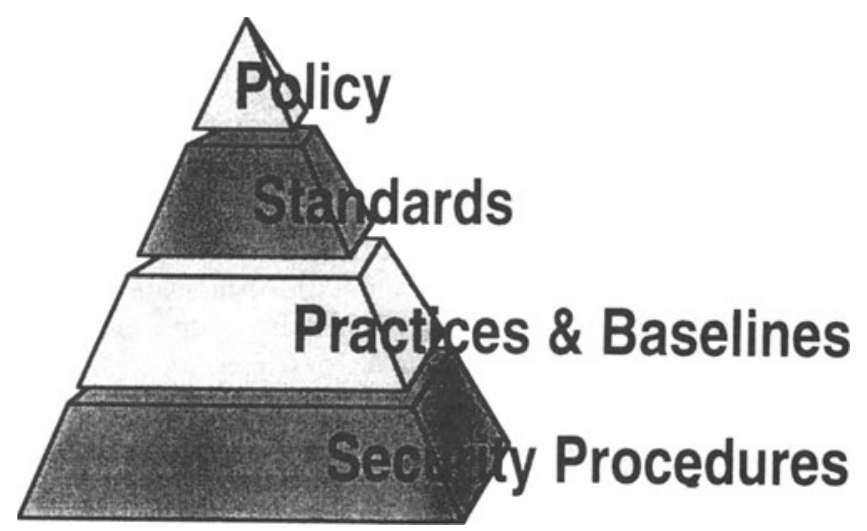

Figure 3. Policies and Procedures 
Electronic access to Highly Confidential or Essential information must not be possible from inside the General Office Area.

- Sensitive Area: Open to individuals with a business need to be in the area, and typically contains assets classified as Confidential or Important that is accessed in clear text. Electronic access to Confidential or Important information will typically be performed from inside a Sensitive Area. Access to this area MUST be logged.

- Highly Sensitive Area: Open to a restricted number of individuals with a business need to be in the area, and typically contains assets classified as Highly Confidential or Essential. Electronic access to Highly Confidential or Essential or Time Critical information will typically be performed from inside a Highly Sensitive Area. Access to this area MUST be logged and strictly enforced (e.g. by use of a turnstile, or the presence of a guard) to prevent 'tailgating'.

For each area, security attributes will be defined such as whether badge access, guard attendance or remote security monitoring is required.

\section{SECURITY MANAGEMENT}

This project aims to provide S.W.I.F.T. with company-wide support, guidance, management and tools so as to efficiently handle day-to-day security administration, to implement security audit recommendations and to effectively manage security incidents. The goal is that, by mid-1997, S.W.I.F.T. will have in place a Global Information Security function, supported by adequate tools and budgets.

Effective security may be seen as a combination of a Strategy (ideas and plans), the tactical elements (solutions and mechanisms) along with administrative and security management functions. This project will improve S.W.I.F.T.'s global security management as well as responsiveness to Security Audit recommendations and put in place the necessary tools to enable effective local and remote monitoring and control of security processes.

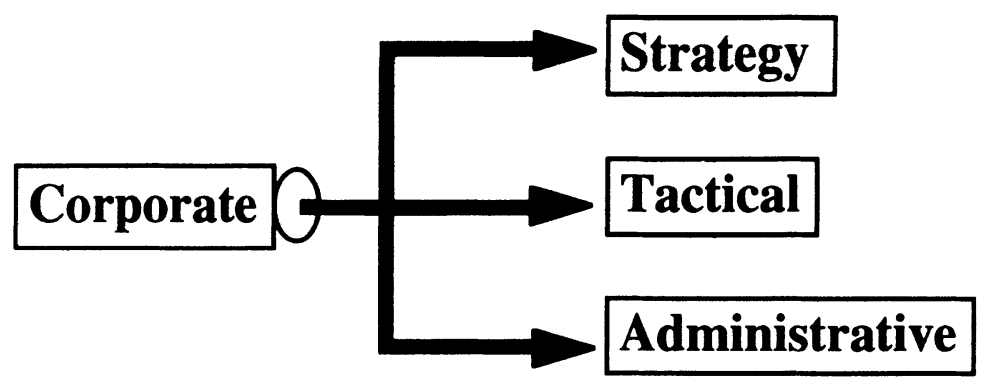

Figure 4. Security Management 


\section{Security Solutions}

Security Solutions will continue to provide security advice and internal services to other departments. The following are planned:

- Review and comment on the security requirements for a variety of projects; provide solutions to security issues and incidents; provide on-going evaluation of security products.

- Roll out and administer a secure Internet infrastructure, with one managed connection per wing/building and an Internet E-mail address for those staff who need it; provide secure support for external Internet access where justified (e.g. via SAP) once adequate controls are in place.

- Improve the audit recommendation closure process; attend monthly coordination meetings with audit/auditee; provide an up-to-date audit recommendations database.

- Continue to provide USE Expertise Centre including: Level 3 USE support, qualification of USE ' 97 facilities; Level 3 support and maintenance for the SMC and perform an impact analysis re ST200/ADM discontinuation.

- Ensure that the USE system is enabled to internally support an RSA key length of 1024-bits (as needed for the Public Key Infrastructure).

\section{Security Administration}

The existing Security Administration function will be strengthened to provide the following services:

- Ongoing security administration functions, based on Service Level Agreements with system owners, and with regular reporting. Provide internal Certification services and act as an internal Registration Authority.

\section{HUMAN BEHAVIOUR AND CULTURE}

This project aims to influence - and to significantly improve - human behaviour within the company with regard to all aspects of security, and to ensure that S.W.I.F.T.'s leadership in this area is highly visible within the international user community. In addition, dedicated security courses (for UNIX and Windows NT) will be provided and relevant staff will have to attend an internal UNIX Security Certification programme.

Security Orientation is part of all staff appraisal programmes. Extensive external promotion of corporate activities is planned and by the end of 1998, S.W.I.F.T. intends to be able to demonstrate a highly visible corporate security culture.

The deliverables under this project will be evident in three distinct areas: 


\section{Internal Security Awareness}

The security culture within S.W.I.F.T. and the intrinsic behaviour of staff must provide a firm foundation for all other security-related activities. S.W.I.F.T. aims to be the best in its field and will therefore implement an effective, highly visible and on-going internal Security Awareness campaign. This will be evident throughout the company in many different ways:

- Internal Publicity: Poster campaigns, regular information sessions, displays of press cuttings, articles in SWIFT World, ISO News and SWIFT Scene, and quarterly updates of our security status in Product News will all serve to reinforce staff awareness of security issues.

- Training: Courses on Managing Information Security will be given to all 'owners' of internal systems.

- Intranet: Dedicated Web pages will be published by GIS on the company Intranet, detailing specific security activities.

- Staff Appraisals: 'Security Orientation' is part of everyone's role profiles and job descriptions and will be a permanent feature of staff appraisals to ensure that every member of staff:

"Recognises that effective security is critical and an essential element of S.W.I.F.T.'s corporate mission." and

"Practises continuous security awareness in all daily activities and takes personal responsibility to act pro-actively upon any and all risks to S.W.I.F.T.'s security (information security, asset protection, loss prevention, business continuity and personal security)."

\section{UNIX and Windows NT Security Courses and UNIX Certification}

With the move towards Open systems and protocols, S.W.I.F.T. wishes to ensure that security is an inherent part of all new products and systems development - from initial concept through to the delivery of secure applications and systems. UNIX and Windows NT are the chosen platforms for most new developments. S.W.I.F.T. plans to train its technical staff in all relevant aspects of UNIX and Windows NT and in the use of appropriate security tools and software methodologies throughout the development process.

Due to the inherent weaknesses of UNIX from the security point of view, a UNIX Security Certification programme has been developed. Starting this year, technical staff who have attended the UNIX Security course, will be subject to a formal examination process, aimed at achieving individual UNIX Security Certification status. Eventually, only those who are Certified will be involved in UNIX-based software development. 


\section{External Awareness of S.W.I.F.T.'s Security Activities}

To increase the external awareness of the many security-related activities in which S.W.I.F.T. is involved and, as a clear demonstration of its leadership in this field, the following are planned:

- SIBOS: A dedicated Security Stream will be established at SIBOS to serve as an international forum on security for the financial community.

- Conferences: Senior staff will give presentations at various international security conferences and seminars.

- Bank Presentations: An 'ABC of Security' presentation will be prepared, and presented to a variety of medium and small institutions worldwide. For large customers, a 'Banks Security Working Group' will be established by S.W.I.F.T., with regular meetings at strategic locations.

- Standards-Making Bodies: S.W.I.F.T. will seek participation in the International Work Groups of those standards-making bodies throughout the world (ESF, ISO, ...) involved with defining security standards. Executive Management will be briefed accordingly.

- Publications: A S.W.I.F.T. Security Guide will be prepared and published to all users as a part of the User Handbook.

\section{APPLICATION SECURITY AND PUBLIC KEY INFRASTRUCTURE}

This project forms one of three initiatives in the area of Tools and Technology - see also System Access Control and Network Segregation. This ambitious and longterm project, commonly known as 'PKI', will enable S.W.I.F.T. to acquire the necessary infrastructure to support very high levels of security within all future products and services.

S.W.I.F.T.'s current security is based on various heterogeneous, ad-hoc solutions which, though making it difficult to be replicated by competitors, are expensive for S.W.I.F.T. to maintain. PKI is a commodity in the Internet world and the availability of that technology is an enabler for competitors to establish cheap and efficient secure communications systems. If we do not implement PKI, others will and S.W.I.F.T. would lose market share.

The FIN security system is designed to protect the S.W.I.F.T. environment from intrusion. It has evolved according to the needs of the traditional correspondent banking environment. It is a one-to-one relationship, in a store-and-forward environement, with S.W.I.F.T. acting as trusted arbitrator. It is not suitable for multi-lateral environments, nor straight user-to-user communications.

By implementing PKI, S.W.I.F.T. will be able to offer:

- true end-to-end message encryption 
- end-to-end message authenticity and non-repudiation by use of digital signatures

- client-to-server session authentication

- systems integrity in terms of deliverables with digital signatures

- network security in the form of secure protection from hostile environments.

These services, based on highly-secure 1024-bit asymmetric cryptography, will provide:

- the basis for a new communications infrastructure, as shown below

- the ability to provide state-of-the-art security for non-FIN messaging services, for internal systems and between the S.W.I.F.T. network(s) and third-party networks

- the basis on which wholly new services can be provided, for example, allowing S.W.I.F.T. to act as the Trusted Third Party for the banks involved in Electronic Commerce activities.

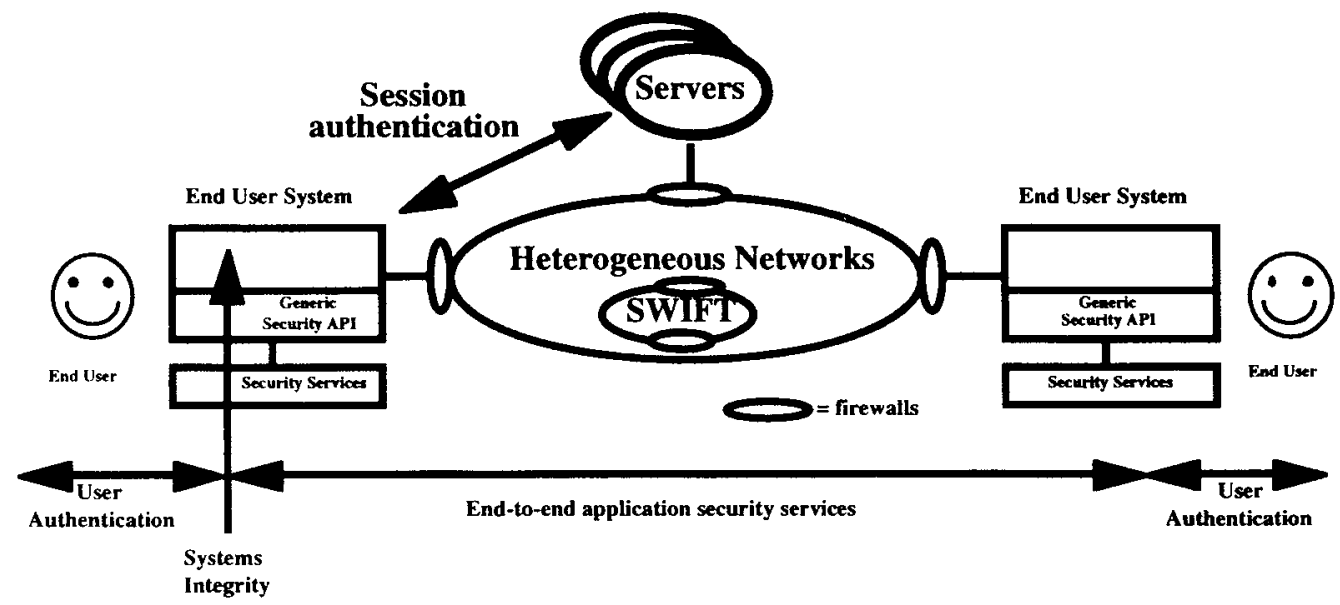

Figure 5. Communications infrastructure

PKI will enable automated and transparent key management, key certification, authentication and non-repudiation services to be offered within each of our customer applications, interfaces and network domains. Security services can then be offered:

- between customer-based interface applications

- between client applications and the network servers

- between networks

- within applications in terms of the authenticity of deliverables, processes and audit trails 
This project focuses on the following areas:

- System Classification Database: to maintain all relevant data for the purposes of managing access controls.

- Logical Access Control Procedures: as a means to securely authorise an end user to gain access to a system.

- System Access Controls: based on two wholly new facilities:

- Security Administration Front End (SAFE) as a means of administering access controls for internal systems

- S.W.I.F.T. Access Server (SAS) as a means of access control for external systems (e.g. for session authentication between remote client applications and network servers).

- Biometrics: using human attributes, such as fingerprint or voice recognition as a secure means of identifying users.

These new facilities will enable a most important part of the new Security Strategy to be achieved, namely to offer:

- Biometrics and/or Smart Cards on our products and on our critical internal workstations to provide the strongest possible means of user authentication.

- Single Sign On for our customers to access our services via the 'S.W.I.F.T. window on the world', based on USE and SAS technology.

- Single Sign On for our internal systems based on biometrics, to drastically reduce the risk of security breaches on our critical internal workstations.

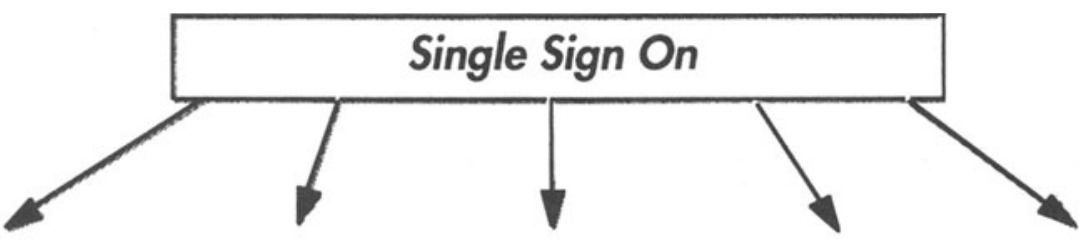

Infomation: Users \& Profiles

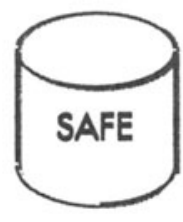

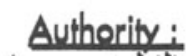
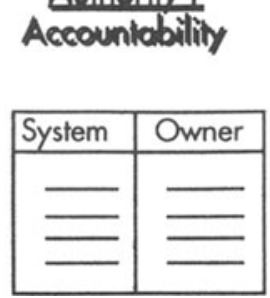

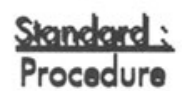

- Password

Administrator

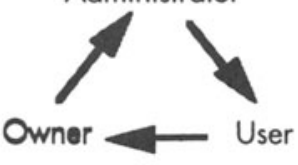

- Token

Authentication:

- Biometrics

Figure 6. Single Sign On 
A centrally-based System Classification Database will be developed, which will store lists of users and user profiles, systems and their owners/administrators, applications, etc., together with detailed access control lists. This information will effectively define precisely who has access to what and on which system. Linked to this, a 'Security Administration Front End' system (SAFE) will be developed, enabling remote management of all network- and application-based access controls.

A software agent (using Kerberos-like techniques) will be included within the security mechanisms embedded within each application, enabling that application to communicate with a central S.W.I.F.T. Access Server, passing on the data obtained from local user-authentication, and being granted permission to allow/disallow application sessions for that user, based on the access control information held centrally.

Providing that the mechanism used for authentication is strong enough, this opens the way for the so-called 'Single Sign On' concept - whereby a user signing on to one system is automatically signed on for all other systems and/or applications ot which that user is entitled.

\section{Use of biometrics}

User identification has traditionally been based either on 'what you know' such as a password, or 'what you own' such as a security token (e.g. smart card).

It is now considered that passwords are no longer sufficiently secure in relation to the critical nature of S.W.I.F.T.'s business and that of its customers. A typical user often has to remember many different passwords for different systems and applications. The human tendency is to simplify things - either by writing down passwords, or by choosing passwords that can be easily recalled (and which are therefore easily guessed). Also, sophisticated tools are now commonly available which, working on the enciphered form, can reveal all but the strongest passwords.

Security tokens are more secure, but can be less user-friendly to use, can be lost / stolen and must be in some way either connected to the workstation or data transferred from the token to the keyboard.

Biometrics, in the form of voice and fingerprint recognition (and also Retina and DNA recognition) have long been too costly to consider as alternatives. However, a recent technological breakthrough has meant that fingerprint recognition is now an affordable option. S.W.I.F.T. plans to take advantage of this and use fingerprint recognition as a strong, reliable and cost-effective means of user identification at the workstation. 


\section{NETWORK SEGREGATION}

This final project in the area of Tools and Technology, coupled with PKI and improves system access controls, will enable S.W.I.F.T. to complete its security architecture for the next millennium.

By separating the S.W.I.F.T. network into separately controlled domains with strong Firewalls at each access point - at the limit of our R\&L domain - the S.W.I.F.T. world can effectively be isolated from the hostile Internet world. Once this is accomplished, S.W.I.F.T. will be able to offer secure TCP/IP connections, and connections via the Internet, both to customers and to remote internal users.

Once again, S.W.I.F.T. aims to be the best and this major investment and reorganisation is seen as fundamental to S.W.I.F.T.'s long-term business strategy. The S.W.I.F.T. networks will be segregated into three distinct domains:

Production domain: the revenue-generating systems

Administrative domain: the systems necessary to administer the production domain Office Automation domain: the internal productivity systems.

These domains will be centrally managed using the secure remote management tools mentioned previously. This new network topology is necessary to be able to take advantage of the new Open technologies in a way which will not threaten the integrity of our business.

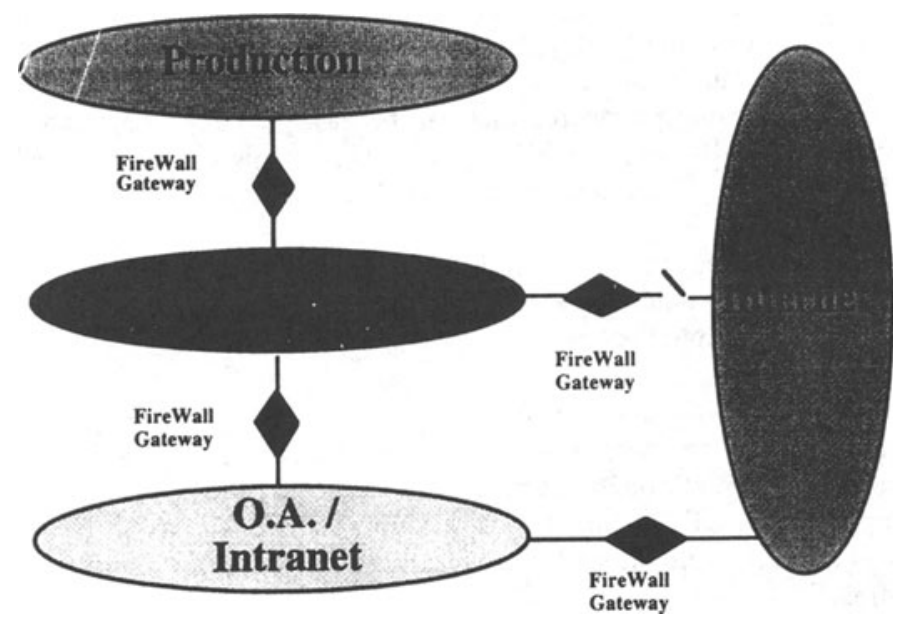

Figure 7. Network segregation 


\section{Firewalls}

The network domains will be isolated from each other and from the external world, by building and implementing appropriate Firewalls. Within each of the networks, data encryption will progressively move towards application-based solutions, taking advantage of the facilities offered by the Public Key Infrastructure project. Dedicated line encryptors will continue to be used to protect sensitive links.

Firewall systems provide intelligent controls right up to the Application level. They are capable of allowing connections only for certain users and to certain applications, while blocking all other attempts at connecting to systems and/or applications for which the user does not have the correct privileges and entitlements. 